\title{
Determinants of physical activity and exercise in healthy older adults: A systematic review
}

Margot A Koeneman ${ }^{1,2^{*}}$, Marieke W Verheijden ${ }^{1,3^{*}}$, Mai J M Chinapaw ${ }^{1,2}$ and Marijke Hopman-Rock ${ }^{1,2,3}$

\begin{abstract}
Background: The health benefits of regular physical activity and exercise have been widely acknowledged. Unfortunately, a decline in physical activity is observed in older adults. Knowledge of the determinants of physical activity (unstructured activity incorporated in daily life) and exercise (structured, planned and repetitive activities) is needed to effectively promote an active lifestyle. Our aim was to systematically review determinants of physical activity and exercise participation among healthy older adults, considering the methodological quality of the included studies.

Methods: Literature searches were conducted in PubMed/Medline and PsycINFO/OVID for peer reviewed manuscripts published in English from 1990 onwards. We included manuscripts that met the following criteria: 1) population: community dwelling healthy older adults, aged 55 and over; 2 ) reporting determinants of physical activity or exercise. The outcome measure was qualified as physical activity, exercise, or combination of the two, measured objectively or using self-report. The methodological quality of the selected studies was examined and a best evidence synthesis was applied to assess the association of the determinants with physical activity or exercise.

Results: Thirty-four manuscripts reporting on 30 studies met the inclusion criteria, of which two were of high methodological quality. Physical activity was reported in four manuscripts, exercise was reported in sixteen and a combination of the two was reported in fourteen manuscripts. Three manuscripts used objective measures, twenty-two manuscripts used self-report measures and nine manuscripts combined a self-report measure with an objective measure. Due to lack of high quality studies and often only one manuscript reporting on a particular determinant, we concluded "insufficient evidence" for most associations between determinants and physical activity or exercise.

Conclusions: Because physical activity was reported in four manuscripts only, the determinants of physical activity particularly need further study. Recommendations for future research include the use of objective measures of physical activity or exercise as well as valid and reliable measures of determinants.
\end{abstract}

Keywords: Prevention, Behaviour change, Determinants, Active lifestyle, Aged

\section{Background}

As the proportion of older adults in Western societies continues to grow, so does their average life expectancy. Even though regular physical activity (PA: unstructured activities incorporated in daily life) and participation in exercise (EX: structured and planned activities) have many health benefits, levels of PA and EX decrease with increasing age [1]. The age related decline in physical capacity is experienced as an increased effort needed to

\footnotetext{
*Correspondence: margot.koeneman@tno.nl; marieke.verheijden@tno.nl 'Body@Work, Research Center for Physical Activity, Work and Health, TNO-VU University Medical Center, Amsterdam, The Netherlands

Full list of author information is available at the end of the article
}

perform daily activities, which could ultimately lead to avoidance of PA and EX [2]. This is a worrisome trend that can be prevented by adopting or maintaining regular PA and EX into old age $[2,3]$. Even when optimal levels of activity cannot be achieved, increasing PA and EX participation can still induce health benefits [1]. The limited success in getting and keeping older adults physically active $[4,5]$ shows a great need for knowledge of determinants of PA and EX.

Recent literature reviews on determinants of PA and EX in older adults have included Randomized Controlled Trials (RCTs) and prospective studies to draw conclusions on causal relationships [6,7]. Martin and Sinden [6]

\section{() Biomed Central}


explored predictors of older adults' adherence to exercise interventions and suggested that different variables predict adherence at different time points, also depending on the type of exercise (e.g. aerobic or strength programs). Adherence to exercise was best predicted by health and health indicators, such as being fit, non-smoking, having an active and healthy lifestyle and psychological determinants, such as high self-efficacy. Van Stralen and colleagues [7] explored determinants of initiation and maintenance of physical activity among older adults and suggested that determinants are partly phase-specific and differ for initiating (up to six months) or maintaining (more than six months) physical activity.

It remains, however, unclear how determinants may differ between PA and EX. Considering the divers nature of physical activity and exercise together with the finding of Martin and Sinden [6] that different types of exercise were predicted by different determinants, it seems plausible that determinants of physical activity and exercise are not the same. We intended to build upon previous work $[6,7]$. In addition, we wanted to differentiate between determinants of PA and EX in healthy older adults only. Therefore, our aim was to assess the determinants of PA as well as EX in healthy older adults, considering the methodological quality of the included studies.

\section{Methods}

\section{Literature search}

We conducted a literature search in the databases PubMed/Medline and PsycINFO/OVID for peer reviewed manuscripts published in English from 1990 onwards. We started the literature searches in May 2009, and conducted updates until January 2011. Search terms included physical activity, exercise and terms describing the target population (e.g., older adults, aged and aging). The full search strategy can be obtained from the corresponding author. Additional searches were performed using reference lists of review articles retrieved through the original search.

\section{Manuscript selection}

Two researchers ( $\mathrm{MK}$ and MV) independently performed an initial selection for eligibility based on the titles of the manuscripts. Three of the authors (MK, $\mathrm{MH}$ and $\mathrm{MC}$ ) independently assessed the remaining abstracts and included manuscripts that met the following criteria: 1) population: community dwelling healthy older adults, aged 55 and over (based on mean age and/ or age range); and 2) prospective manuscripts reporting determinants of PA or EX. International guidelines [3] do not provide a clear cut point for "old age". We therefore arbitrarily chose to include studies with a population aged 55 and older. Manuscripts reporting on a specific subsample of the older population (e.g. ethnicity,
IQ, confined geographic area or diagnosed diseases) were excluded. Similarly, manuscripts reporting on interventions to prevent falls or obesity were excluded.

\section{Categorisation}

Manuscripts were categorised based on: outcome measure (PA or EX, and objective or self-report). We used the definition by Caspersen et al. [8] to categorise the outcome measure as either PA or EX. PA was defined as 'occupational physical activity, household activities and walking/ strolling for entertainment, social goals or transport'. EX was defined as 'physical activity, which is planned, structured, and repetitive, with the specific goal to maintain or improve physical fitness'. In line with this categorisation, walking can either be categorised as PA, when participants walk as a form of transport, or as EX, when participants are instructed to have planned, repetitive walks of specific duration. The outcome measure was further categorised as either objective (such as: pedometers and accelerometers) or self-report (such as: exercise logs, questionnaires and recording of class attendance).

\section{Methodological quality assessment and evidence synthesis}

The methodological quality of the selected studies was assessed based on the items on validity and precision derived from a checklist from Chinapaw et al., Brown et al. and Uijtdewilligen et al. [9-11]. The checklist is presented in Table 1 and consists of 8 items describing four categories (study population and participation; study attrition; data collection; and data analyses). Two authors (MK, MV) and a trained statistician independently appraised the methodological quality of the included studies. Items were rated "+" if the requested information was present in the paper and criteria were met. Items were rated '-' if the requested information was present in the paper and criteria were not met. Items were scored "?" if insufficient information was provided. If one of the selected manuscripts referred to another publication for information on the checklist items, this publication was retrieved and the information was extracted. Disagreement was resolved through discussion. The overall methodological quality of a study is expressed as a percentage calculated from the total number of "+" scores divided by 8 . Manuscripts with a percentage of $70 \%$ or more were considered high quality [11].

Because of the heterogeneity of the methods used in the included manuscripts statistical pooling of data was not possible. Instead, we applied a best evidence synthesis similar to Chinapaw et al. and Uijtdewilligen et al. $[9,11]$. This synthesis takes the methodological quality, the number of studies and the consistency of the evidence into account. 
Table 1 Criteria list for assessment of the methodological quality of prospective studies

Criteria:
Study population and participation (baseline): The study sample
represents the population of interest on key characteristics

(rating of criteria: ' + ' = yes, ' '- = no, '?' = not or insufficiently described)

1. Participation rate at baseline at least $80 \%$, or if the non-response was not selective (show that baseline study sample does not significantly differ from population of eligible subjects)

Study attrition: Loss to follow-up is not associated with key characteristics (i.e. the study data adequately represent the sample)

2. Response at short-term follow-up (up to 12 months) was at least $80 \%$ of the number of participants at baseline and response at long-term follow-up was at least $70 \%$ of the number of participants at baseline
3. Not selective non-response during follow-up measurement(s) a ' $t$ ' is given only if non-selective dropout on key characteristics (age, gender, relevant determinants, and outcome measure) is reported in the text or tables

Data collection:

4. Adequate measurement of PA, EX or PA/EX: objective measurement and not by self-report (self-report $=$ ' - , no/insufficient information $=$ '?')

5. Determinants of PA or EX behaviour were measured with a reliable tool ' $t$ ' is given only if measures of determinants showed ICC/KAPPA of $\geq 0.60$ (or Pearson correlation above .70) assessed within the appropriate target population. For biological variables, a ' $t$ ' was given only if a standardised protocol was followed, and trained researchers assessed the determinants. A ' $t$ ' was also given for age, gender, ethnicity, marital status, socio-economic status, employment status, education, income, intervention condition and objective assessment of environmental characteristics (proximity of parks and weather conditions). Expressed as the number of cases in which the measurement of determinants were scored + , divided by the total number of determinants measured in all studies.

6. Determinants of PA or EX behaviour were measured with a valid tool ' + ' is given only if measures of determinants showed correlations of $\geq 0.40$ with other similar constructs measured within the appropriate target population. For PA, EX or PA/EX variables (e.g. past physical activity), a ' + ' was given only if variables were assessed with an objective measurement instrument (e.g. accelerometer/pedometer). For biological variables, a ' + ' was given only if a standardised protocol was followed, and trained researchers assessed the determinants. A ' + ' was also given for age, gender, ethnicity, marital status, socio-economic status, employment status, education, income, intervention condition and objective assessment of environmental characteristics (proximity of parks and weather conditions). Expressed as the number of cases in which the measurement of determinants were scored + , divided by the total number of determinants measured in all studies.

Data analyses:

7. The statistical model used was appropriate

8. The number of cases was at least 10 times the number of the independent variables
The evidence for the relationship between determinants and behavioural outcome (PA, EX or PA/EX) was rated strong (consistent findings in multiple $(\geq 2)$ high methodological quality studies), moderate (consistent findings in one high quality and at least one low methodological quality studies or consistent findings in multiple $(\geq 2)$ low methodological quality studies) or as insufficient (only one study available or inconsistent findings in multiple ( $\geq 2$ ) studies). Results were considered to be consistent when at least $75 \%$ of the studies showed significant results in the same direction. When at least $75 \%$ of the studies found no significant association this would be considered insufficient evidence for an association ("no evidence for an association").

\section{Data extraction}

For each individual manuscript, determinants were categorized as ' + ' (having a significant positive association with PA/EX), '-' (having a significant negative association with PA/EX), or '0' (having no association with PA/EX). Significance levels were set at $\mathrm{p}<0.05$. Notably, when manuscripts were reviewed, it appeared that multiple manuscripts reported on the same original data set. These clusters of manuscripts were made identifiable in Figure 1 and Table 2 and 3. If separate manuscripts reporting on the same study described similar associations, they represent a single count in the evidence synthesis. If separate manuscripts reporting on the same study described different associations, they each represent a count in the evidence synthesis.

\section{Results}

The initial search retrieved 8278 manuscripts (see flowchart in Figure 1). Thirty-four manuscripts reporting on 30 studies, met the inclusion criteria (19 manuscripts obtained from PubMed/Medline, 13 manuscripts from PsycINFO/OVID and 2 manuscripts through the reference lists of reviews retrieved in the search). Of the 


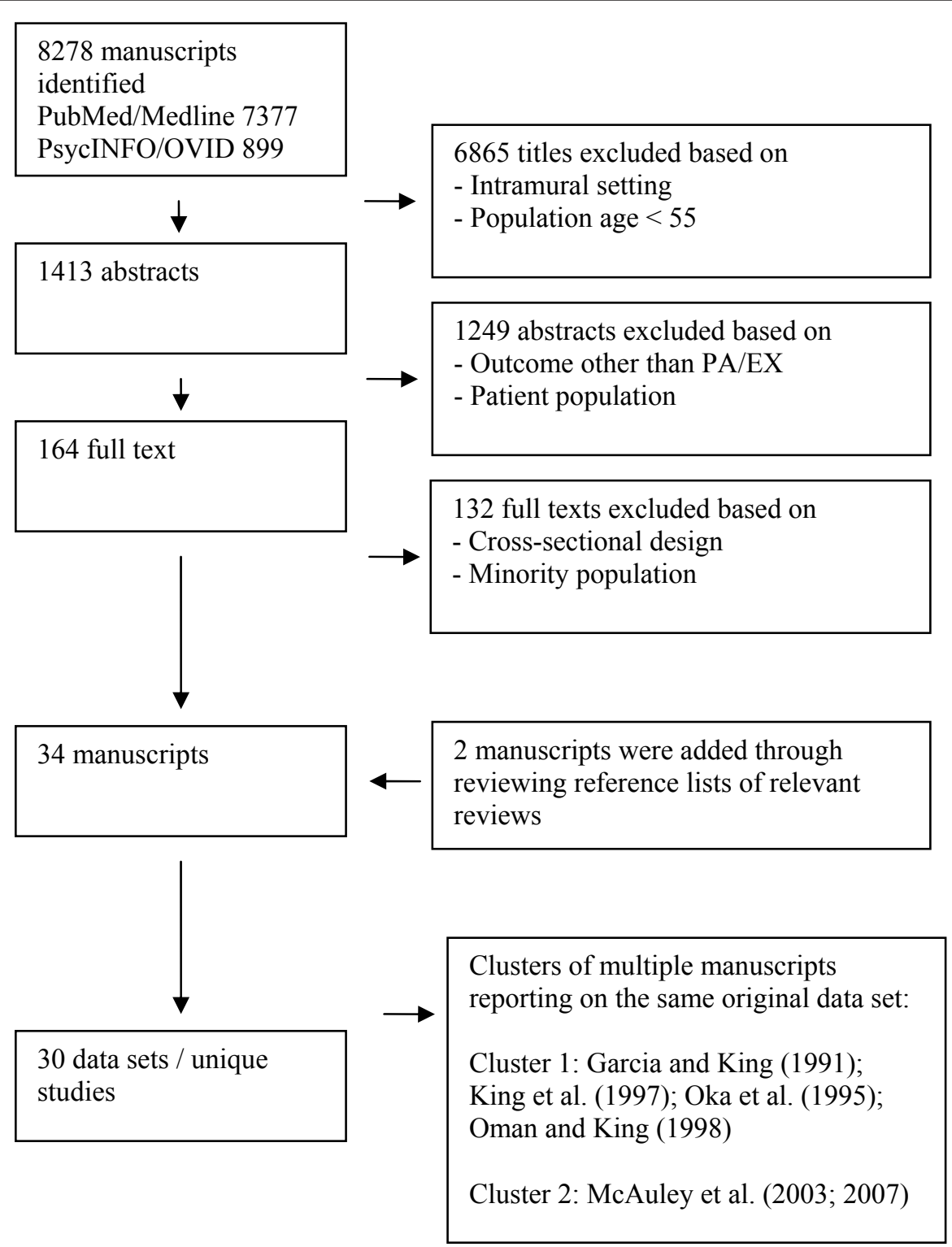

Figure 1 Flowchart of study selection process

included manuscripts, 12 were observational [12-23], 14 were intervention [24-37] and 8 were RCTs [38-45]. Table 2 presents the characteristics of the included manuscripts.

\section{Outcome measure}

PA was reported in four manuscripts [12,15,23,35]. EX was reported in 16 manuscripts [22,24,25,28,29,31-34, $36-38,40,42,43,45]$ and a combination of PA and EX (PA/EX) was reported in 14 manuscripts [13,14,16-21, $26,27,30,39,41,44]$.

Three out of the 34 manuscripts objectively measured PA or PA/EX using pedometers or accelerometers $[23,35,41]$. Twenty-two manuscripts used self-report measures, such as questionnaires, exercise logs or class attendance $\operatorname{logs}$ completed by the participants [12-22,24,26,27,29,30,34,37-39,43,44]. In nine manuscripts a self-report measure was combined with validation through class attendance recorded by the instructor and/or data derived from a pedometer/accelerometer/ oxygen uptake ([25,28,31-33,36,40,42,45]; see Table 2).

\section{Methodological quality assessment}

The methodological quality scores are presented in Table 3. Initial disagreement (25\%) between the raters was based on differences in reading and interpretation. The majority of the initial disagreement was based on items 5 and 6 (were the determinants of PA or EX 
Table 2 Manuscripts reporting on determinants of physical activity and exercise in healthy older adults

\begin{tabular}{|c|c|c|c|}
\hline Reference & $\begin{array}{l}\text { Design: Intervention study, } \\
\text { observational study or } \\
\text { randomized controlled trial } \\
\text { Age: Mean (SD) or [Range] } \\
\text { left } \\
\text { Quality score: \% }\end{array}$ & $\begin{array}{l}\text { Intervention: Content } \\
\text { Outcome measure: Self-report or objective + } \\
\text { instrument } \\
\text { Outcome: unstructured physical activity (PA), } \\
\text { structured exercise (EX) or a combination of } \\
\text { physical activity and exercise (PA/EX) - for } \\
\text { definitions see main text }\end{array}$ & $\begin{array}{l}\text { Determinants: Positive } \\
\text { association (+), negative } \\
\text { association (-) or no } \\
\text { association (NS) }\end{array}$ \\
\hline $\begin{array}{l}\text { [24] Boyette } \\
\text { et al. } \\
1997 \\
\text { U.S. }\end{array}$ & $\begin{array}{l}\text { Design: } \\
\text { Intervention study } \\
\text { Age: } 71.3(4.6) \\
\text { Quality score: } 29\end{array}$ & $\begin{array}{l}\text { Intervention: } \\
\text { Strength training intervention with considerable } \\
\text { support from staff and program organization aimed at } \\
\text { convenience } \\
\text { Outcome measure: } \\
\text { Self-report } \\
\text { PEP } \\
\text { Outcome: } \\
\text { "Do you exercise for muscular strength and } \\
\text { endurance?"/"Do you exercise for flexibility? (EX) }\end{array}$ & $\begin{array}{l}\text { Satisfaction with exercise routine } \\
(+) \\
\text { Satisfaction with body image (+) } \\
\text { Age (NS) } \\
\text { Weight (NS) } \\
\text { BMI (NS) } \\
\text { Easy Access to exercise location } \\
\text { (NS) }\end{array}$ \\
\hline $\begin{array}{l}{[25]} \\
\text { Brassington } \\
\text { et al. } \\
2002 \\
\text { U.S. }\end{array}$ & $\begin{array}{l}\text { Design: Intervention study } \\
\text { Age: } 70.2(4.1) \\
\text { Quality score: } 38\end{array}$ & $\begin{array}{l}\text { Intervention: } \\
\text { Endurance and strengthening exercise prescription or } \\
\text { stretching and flexibility exercise prescription } \\
\text { combined with telephone counselling to overcome } \\
\text { exercise barriers for the promotion of exercise } \\
\text { adherence } \\
\text { Outcome measure: } \\
\text { Self-report } \\
\text { Exercise logs, validation through class-attendance and } \\
\text { 'vitalogs' } \\
\text { Outcome: } \\
\text { Adherence to prescribed exercise (EX) }\end{array}$ & $\begin{array}{l}\text { Change in exercise self-efficacy } \\
(+) \\
\text { Self-reported fitness outcome } \\
\text { realizations (+) } \\
\text { Baseline exercise self-efficacy (NS) } \\
\text { Baseline fitness outcome } \\
\text { expectancies (NS) } \\
\text { Baseline psychological outcome } \\
\text { expectancies (NS) } \\
\text { Baseline social support for } \\
\text { exercise (NS) } \\
\text { Change in social support (NS) }\end{array}$ \\
\hline $\begin{array}{l}\text { [12] Burton } \\
\text { et al. } \\
1999 \\
\text { U.S. }\end{array}$ & $\begin{array}{l}\text { Design:Observational study } \\
\text { Age: }\left[65-85^{+}\right] \\
\text {Quality score: } 49\end{array}$ & $\begin{array}{l}\text { Intervention: } \\
\text { Not applicable } \\
\text { Outcome measure: } \\
\text { Self-report } \\
\text { 'how often do you perform physical activity such as } \\
\text { walking briskly, gardening, or heavy housework?' } \\
\text { Outcome: } \\
\text { Performing brisk physical activity at least three times a } \\
\text { week = active (less is considered sedentary or } \\
\text { insufficiently active) (PA) }\end{array}$ & $\begin{array}{l}\text { Age }(-) \\
\text { Gender (male) }(+) \\
\text { Marital status }(+) \\
\text { General physical health }(+) \\
\text { Belief in importance of physical } \\
\text { activity (+) } \\
\text { Emotional distress (-) } \\
\text { Advice of physician about } \\
\text { getting more exercise (-) } \\
\text { Ethnicity (NS) } \\
\text { Education (NS) } \\
\text { Self-mastery (NS) } \\
\text { Having a confidant (NS) }\end{array}$ \\
\hline $\begin{array}{l}\text { [38] } \\
\text { Cheung et } \\
\text { al. } \\
\text { 2006 } \\
\text { U.S. }\end{array}$ & $\begin{array}{l}\text { Design: Randomized } \\
\text { controlled trial } \\
\text { Age: } 75.1(7.0) \\
\text { Quality score: } 25\end{array}$ & $\begin{array}{l}\text { Intervention: } \\
\text { Walking prescription and instruction with weekly } \\
\text { prompting by nurse or computer } \\
\text { Outcome measure: } \\
\text { Self-report } \\
\text { Exercise logs walking behaviour } \\
\text { Outcome: } \\
\text { Walking behaviour (miles, minutes and perceived } \\
\text { exertion) and adherence to prescription (EX) }\end{array}$ & $\begin{array}{l}\text { Exercise self-efficacy }(+) \\
\text { Stage of change (NS) }\end{array}$ \\
\hline $\begin{array}{l}{[39]} \\
\text { Costanzo } \\
\text { and Walker } \\
2008 \\
\text { U.S }\end{array}$ & $\begin{array}{l}\text { Design: Randomized } \\
\text { controlled trial } \\
\text { Age: [50-65] } \\
\text { Quality score: } 50\end{array}$ & $\begin{array}{l}\text { Intervention: } \\
\text { Behavioural counselling to increase exercise self- } \\
\text { efficacy and social support from friends and family } \\
\text { Outcome measure: } \\
\text { Self-report } \\
\text { Modified 7-day activity recall } \\
\text { Outcome: } \\
\text { Self-reported moderate or greater physical activities of } \\
\text { at least } 4 \text { MET reported in minutes based on Modified } \\
7 \text {-day activity interview, listing several common } \\
\text { activities: "moderate" (eg, cleaning, fishing, raking); } \\
\text { "hard" activities (eg, golf, scrubbing); and "very hard" } \\
\text { (eg, swimming) (PA/EX) }\end{array}$ & $\begin{array}{l}\text { Change in exercise self-efficacy } \\
(+) \\
\text { Change in social support (family) } \\
(-) \\
\text { Change in social support (friends) } \\
\text { (NS) }\end{array}$ \\
\hline
\end{tabular}


Table 2 Manuscripts reporting on determinants of physical activity and exercise in healthy older adults (Continued)

\begin{tabular}{|c|c|c|c|c|}
\hline \multirow[t]{4}{*}{ Cluster $1 *$} & $\begin{array}{l}{[42]} \\
\text { Garcia } \\
\text { and King } \\
1991 \\
\text { U.S. }\end{array}$ & $\begin{array}{l}\text { Design: Randomized } \\
\text { controlled trial } \\
\text { Age: } 56.4(4.2) \\
\text { Quality score: } 41\end{array}$ & $\begin{array}{l}\text { Intervention: } \\
\text { Personalized exercise prescription class-based or } \\
\text { home-based (home-based included regular telephone } \\
\text { contact) } \\
\text { Outcome measure: } \\
\text { Self-report } \\
\text { Exercise logs validation through 'vitalogs' } \\
\text { Outcome: } \\
\text { Exercise performed relative to prescription (EX) }\end{array}$ & $\begin{array}{l}\text { Marital status (+) } \\
\text { Smoking status (-) } \\
\text { Exercise self-efficacy (+) } \\
\text { Exercise intervention condition } \\
\text { (home-based vs class-based) (+) } \\
\text { Age (NS) } \\
\text { Gender (NS) } \\
\text { Ethnicity (NS) } \\
\text { Education (NS) } \\
\text { Income (NS) } \\
\text { BMI (NS) } \\
\text { Self-motivation (NS) } \\
\text { Exercise intervention condition } \\
\text { (low vs high intensity) (NS) } \\
\text { Perceived exertion, enjoyment } \\
\text { and convenience of intervention } \\
\text { (NS) }\end{array}$ \\
\hline & $\begin{array}{l}{[28] \text { King }} \\
\text { et al. } \\
1997 \\
\text { U.S. }\end{array}$ & $\begin{array}{l}\text { Design: Intervention study } \\
\text { Age: [50-65] } \\
\text { Quality score: } 40\end{array}$ & $\begin{array}{l}\text { Intervention: } \\
\text { Personalized exercise prescription class-based or } \\
\text { home- based (home-based included regular telephone } \\
\text { contact) } \\
\text { Outcome measure: } \\
\text { Self-report } \\
\text { Exercise logs validation through 'vitalogs' } \\
\text { Outcome: } \\
\text { Exercise adherence to at least two thirds of exercise } \\
\text { prescription (EX) }\end{array}$ & $\begin{array}{l}\text { Exercise intervention condition } \\
\text { (home-based vs class-based) (+) } \\
\text { Stress }(-) \\
\text { General physical functioning (+) } \\
\text { Education (-) } \\
\text { BMI (-) } \\
\text { Age (NS) } \\
\text { Gender (NS) } \\
\text { Marital status (NS) } \\
\text { Employment status (NS) } \\
\text { Social support (NS) }\end{array}$ \\
\hline & $\begin{array}{l}{[45] \text { Oka }} \\
\text { et al. } \\
1995 \\
\text { U.S. }\end{array}$ & $\begin{array}{l}\text { Design: Randomized } \\
\text { controlled trial } \\
\text { Age: [50-65] } \\
\text { Quality score: } 21\end{array}$ & $\begin{array}{l}\text { Intervention: } \\
\text { Personalized exercise prescription group-based or } \\
\text { home- based (home-based included telephone } \\
\text { counselling related to meeting exercise prescription) } \\
\text { Outcome measure: } \\
\text { Self-report } \\
\text { Exercise logs validation through 'vitalogs' } \\
\text { Outcome: } \\
\text { Exercise adherence as percentage of exercise } \\
\text { prescription (EX) }\end{array}$ & $\begin{array}{l}\text { Smoking status (-) } \\
\text { Exercise intervention condition } \\
\text { (home-based vs class-based) (+) } \\
\text { Preferring a lesser amount of } \\
\text { social support from staff (+) } \\
\text { Initial and continued social } \\
\text { support (friends/family/staff) (+) } \\
\text { Family satisfaction (-) } \\
\text { Gender (NS) }\end{array}$ \\
\hline & $\begin{array}{l}{[32]} \\
\text { Oman } \\
\text { and King } \\
1998 \\
\text { U.S. }\end{array}$ & $\begin{array}{l}\text { Design: Intervention study } \\
\text { Age: } 56.2(4.2) \\
\text { Quality score: } 38\end{array}$ & $\begin{array}{l}\text { Intervention: } \\
\text { Personalized exercise prescription class-based or } \\
\text { home-based (home-based included telephone } \\
\text { counselling related to meeting exercise prescription) } \\
\text { Outcome measure: } \\
\text { Self-report } \\
\text { Exercise logs validation through 'vitalogs' } \\
\text { Outcome: } \\
\text { Exercise performed relative to prescription (EX) }\end{array}$ & $\begin{array}{l}\text { Exercise self-efficacy (+) } \\
\text { Exercise intervention condition } \\
\text { (home-based vs class-based) (+) } \\
\text { Prior exercise adherence (+) } \\
\text { Exercise intervention intensity } \\
\text { (NS) } \\
\text { Exercise self-efficacy (+) } \\
\text { Affect (NS) }\end{array}$ \\
\hline \multirow[t]{2}{*}{ Cluster $2^{*}$} & $\begin{array}{l}{[44]} \\
\text { McAuley } \\
\text { et al. } \\
2003 \\
\text { U.S. }\end{array}$ & $\begin{array}{l}\text { Design: Randomized } \\
\text { controlled trial } \\
\text { Age: } 66.7(5.4) \\
\text { Quality score: } 38\end{array}$ & $\begin{array}{l}\text { Intervention: } \\
\text { Exercise classes three times a week either walking or a } \\
\text { stretching and toning program } \\
\text { Outcome measure: } \\
\text { Self-report } \\
\text { PASE (including leisure, household, and occupational } \\
\text { activity) } \\
\text { Outcome: } \\
\text { Level of activity based on PASE (PA/EX) }\end{array}$ & $\begin{array}{l}\text { Social support (NS) } \\
\text { Prior exercise adherence (NS) } \\
\text { Level of physical activity at } 2 \\
\text { years after intervention (+) }\end{array}$ \\
\hline & $\begin{array}{l}{[30]} \\
\text { McAuley } \\
\text { et al. } \\
\text { 2007 } \\
\text { U.S. }\end{array}$ & $\begin{array}{l}\text { Design: Intervention study } \\
\text { Age: } 66.7(5.4) \\
\text { Quality score: } 56\end{array}$ & $\begin{array}{l}\text { Intervention: } \\
\text { Exercise classes three times a week either walking or a } \\
\text { stretching and toning program } \\
\text { Outcome measure: } \\
\text { Self-report } \\
\text { PASE (including leisure, household, and occupational } \\
\text { activity) } \\
\text { Outcome: } \\
\text { Level of activity based on PASE (PA/EX) }\end{array}$ & $\begin{array}{l}\text { Positive affect } 2 \text { years after } \\
\text { intervention (+) } \\
\text { Exercise self-efficacy } 2 \text { years after } \\
\text { intervention (+) } \\
\text { Exercise intervention condition } \\
\text { (Walking vs Stretching) (+) } \\
\text { Age (NS) } \\
\text { Gender (NS) } \\
\text { Ethnicity (NS) } \\
\text { Marital status (NS) } \\
\text { Education (NS) } \\
\text { Income (NS) }\end{array}$ \\
\hline
\end{tabular}


Table 2 Manuscripts reporting on determinants of physical activity and exercise in healthy older adults (Continued)

\begin{tabular}{ll}
\hline$[40]$ & Design: Randomized \\
Dubbert et & controlled trial \\
al. & Age: $68.7(4.7)$ \\
2002 & Quality score: 41
\end{tabular}

US.
Quality score: 4
Intervention:

Instruction video and individualized walking program with prompting phone calls or personalized phone

calls with nurse counselling following stage of change

Outcome measure:

Self-report

Walking diary, validation through peers and

accelerometers with additional assessment using the

7-day Physical Activity Recall (PAR)

Outcome:

Episodes of at least 10 minutes duration walking for exercise (EX)

[26] Emery

et al.

1992

U.S.

Design: Intervention study
Age: $67.0(4.9)$
Quality score: 19

Intervention:

for 4 months), a yoga control or a waiting list control group
Aerobics exercise group (3 supervised exercise sessions

\section{Outcome measure:}

Self-report

Retrospective self-report of physical activity during

prior 12 months

Outcome:

Number of months, days per month, and minutes per

day of physical activity (measure for overall activity

and specific activities for which the participants were trained) converted to energy expenditure (PA/EX)

\begin{tabular}{ll}
\hline$[41]$ & Design: Randomized \\
Finkelstein & controlled trial \\
et al. & Age: $[50-85]$ \\
2008 & Quality score: 63 \\
U.S. &
\end{tabular}

U.S.

\section{Intervention:}

Financial incentive for minutes walking, jogging or running

\section{Outcome measure:}

Objective

Pedometer (with paper back-up logs in case of technical problems)

Outcome:

Minutes logged walking, jogging or running (PA/EX)

\begin{tabular}{ll}
\hline$[13]$ & Design: Observational study \\
Hirvensalo & Age: [65-84] \\
et al. & Quality score: 19
\end{tabular}

2000

Finland

Intervention:

Outcome measure:

Self-report

The level of physical activity and its intensity was assessed using a six-point scale: 1) moving only in connection with necessary chores, 2) walking or other outdoor activities 1-2 times/week, 3) walking or other outdoors activities several times/week, 4) 1-2 times/ week to the point of perspiring and heavy breathing, 5) several times/week to the point of perspiring and heavy breathing, 6) keep-fit exercise or competitive sport several times a week.

Outcome:

Level of physical activity based on categories of 6 point scale (PA/EX)

[27] Jancey

et al.

2007

Design: Intervention study

Age: [65-74]

Quality score: 39

\section{Intervention:}

Prescription walking intervention (aerobic, balance, strength and flexibility components), with trained walk leaders, offering advice, reassurance, encouragement, feedback and health education. Including nonmonetary incentives and gatherings to enhance social support

Outcome measure:

Self-report

IPAQ

\section{Outcome:}

Level of activity based on IPAQ (including occupational, household and leisure activity) (PA/EX)
Walking companion (+)

BMI (reduction) $(+)$

Change in mobility

(improvement) (+)

Change in general physical

health (improvement) (+)

Exercise intervention condition

(phone calls vs. no phone calls)

$(+)$

Smoking status (NS)

Change in general mental health (NS)

Cardiorespiratory endurance (+)

Motor speed (+)

Anxiety (-)

Age (NS)

Gender (NS)

Prior exercise adherence (NS) General mental health (NS)

General cognitive functioning (NS)

\section{Education (-)}

Income (-)

Employment status (-)

Exercise intervention condition

(financial incentive vs. no-

incentive) $(+)$

Age (NS)

Gender (NS)

\section{Age (-)}

Cardiovascular/musculoskeletal diseases (-)

Competitive sports early in life $(+)$

Recreations sports in adulthood $(+)$

Socioeconomic status $(+)$

BMI (-)

Loneliness (-)

Walking self-efficacy (-)

Baseline activity level $(+)$

Age (NS)

Gender (NS) 
Table 2 Manuscripts reporting on determinants of physical activity and exercise in healthy older adults (Continued)

\begin{tabular}{lll}
\hline [14] Kahana & Design: Observational study & Intervention: \\
et al. & Age: $79.1(3.1)$ & Not applicable \\
2005 & Quality score: 38 & Outcome measure: \\
U.S. & Self-report \\
& The total number of hours per week: walking, \\
& swimming, golfing, running/jogging, aerobics, \\
& stretching or calisthenics, weight lifting, dancing, \\
& biking, and other exercises. \\
& Outcome: \\
& The total number of hours per week (PA/EX) \\
\end{tabular}

\begin{tabular}{lll}
\hline [43] Li et al. & Design Randomized controlled & Intervention: \\
2001 & trial & Tai Chi practice sessions twice a week \\
U.S. & Age: $72.8(5.1)$ & Outcome measure: \\
& Quality score: 25 & Class-attendance recorded by instructor \\
& Outcome: \\
& Participants practise session attendance (EX) \\
\hline
\end{tabular}

\begin{tabular}{ll}
\hline [15] Li et al. & Design: Observational study \\
2005 & Age: $73.9(2.6)$ \\
U.S. & Quality score: 47
\end{tabular}

Intervention:

\section{Intervention:
Not applicable}

Outcome measure:

Self-report

Neighbourhood walking (walking or strolling though neighbourhood, walked or done physical activities with neighbours or gone to the park for walks or other physical activities)

Outcome:

Neighbourhood walking over the past 12 months (PA)

\begin{tabular}{ll}
\hline [29] Lucidi & Design: Intervention study \\
et al. & Age: $[65-90]$ \\
2006 & Quality score: 50
\end{tabular}

Italy

Intervention:

Sport activity program with two sessions per week including aerobic exercise, strength training, balance and flexibility

Outcome measure:

Self-report

Class-attendance recorded by instructor

Gender (male) (-)

Future orientation (+)

Age (NS)

General physical health (NS)

Outcome:

Percentage of attended sessions divided by number of possible sessions over the three months of exercise classes (EX)

\begin{tabular}{|c|c|c|}
\hline & & $\begin{array}{l}\text { Outcome: } \\
\text { Percentage of attended sessions divided by number of } \\
\text { possible sessions over the three months of exercise } \\
\text { classes (EX) }\end{array}$ \\
\hline $\begin{array}{l}\text { [16] Michael } \\
\text { et al. } \\
2010 \\
\text { U.S. }\end{array}$ & $\begin{array}{l}\text { Design: Observational study } \\
\text { Age: }\left[65^{+}\right] \\
\text {Quality score: } 75\end{array}$ & $\begin{array}{l}\text { Intervention: } \\
\text { Not applicable } \\
\text { Outcome measure: } \\
\text { Self-report } \\
2 \text { questions of the PASE } \\
\text { "Over the past } 7 \text { days, how often did you walk outside } \\
\text { your home or yard for any reason. For example for fun } \\
\text { or exercise, walking to work, walking the dog, etc.?" } \\
\text { "On average, how many hours per day did you spend } \\
\text { walking?" } \\
\text { Outcome: } \\
\text { Walking behaviour (PA/EX) }\end{array}$ \\
\hline
\end{tabular}

Change in exercise self-efficacy

$(+)$

Neighbourhood safety for

walking (+)

Access to exercise facilities (+)

Education (-)

Neighbourhood social cohesion (NS)

Exercise self-efficacy (NS)

General physical health (NS)

Income (NS)

Intention (+)

Perceived behavioural control

(NS)

Exercise self-efficacy (NS)

Exercise attitude (NS)

Subjective norm (NS)

\begin{tabular}{|c|c|c|c|}
\hline $\begin{array}{l}\text { [31] Morey } \\
\text { et al. } \\
2003 \\
\text { U.S. }\end{array}$ & $\begin{array}{l}\text { Design: Intervention study } \\
\text { Age: } 71.5 \text { (4.9) } \\
\text { Quality score: } 32\end{array}$ & $\begin{array}{l}\text { Intervention: } \\
\text { Exercise intervention to improve physical functioning } \\
\text { supervised phase (followed by home-based phase for } \\
\text { one of the two randomized groups) } \\
\text { Outcome measure: } \\
\text { Self-report validation through measured oxygen } \\
\text { uptake } \\
\text { Outcome: } \\
\text { Following exercise prescription (EX). Participants were } \\
\text { classified as adherent if their exercise averaged } 20 \\
\text { minutes or more, } 3 \text { or more days a week, over six } \\
\text { months }\end{array}$ & $\begin{array}{l}\text { Gender (male) (+) } \\
\text { Depression (-) } \\
\text { BMI (-) } \\
\text { General physical functioning (+) } \\
\text { Chronic diseases (-) } \\
\text { Pain (-) } \\
\text { Prior exercise adherence } \\
\text { (weekend exercise home-work) } \\
(+) \\
\text { Age (NS) } \\
\text { Ethnicity (NS) } \\
\text { General physical health (NS) } \\
\text { Exercise self-efficacy (NS) } \\
\text { Locus of Control (NS) } \\
\text { Social support (NS) } \\
\text { Exercise intervention condition } \\
\text { (aerobic only vs aerobic }+ \\
\text { flexibility) (NS) }\end{array}$ \\
\hline
\end{tabular}


Table 2 Manuscripts reporting on determinants of physical activity and exercise in healthy older adults (Continued)

[17] Nitz and Choy 2007

Australia
Design: Observational study

Age: [40-80]

Quality score: 15
Intervention:

Not applicable

Outcome measure:

Self-report

The level of physical activity and its intensity was assessed using a six-point scale: 1) moving only in connection with necessary chores, 2) walking or other outdoor activities 1-2 times/week, 3) walking or other outdoors activities several times/week, 4) 1-2 times/ week to the point of perspiring and heavy breathing, 5) several times/week to the point of perspiring and heavy breathing, 6) keep-fit exercise or competitive sport several times a week.

Outcome:

Level of physical activity based on categories of 6 point scale (PA/EX)

\begin{tabular}{ll}
\hline [33] Oman & Design: Intervention study \\
and King & Age: $65.5(4.3)$ \\
2000 & Quality score: 25 \\
U.S. &
\end{tabular}

\section{Intervention:}

Personalized exercise prescription class-based or home-based (home-based included telephone

counselling related to meeting exercise prescription)
BMI (-)

Baseline activity level (+)

Change in BMI (NS)

Age (NS)

Number of falls (NS)

Number of falls since baseline (NS)

Stability (NS)

Number of medical conditions

(NS)

Increase in number of medical conditions (NS)

Number of medications (NS)

Change in number of medications (NS)

Major life events (-)

Outcome measure:

Exercise logs validation through 'vitalogs'

\section{Outcome:}

Exercise performed relative to prescription (EX)

\begin{tabular}{|c|c|c|c|}
\hline $\begin{array}{l}\text { [34] Rhodes } \\
\text { et al. } \\
2001 \\
\text { Canada }\end{array}$ & $\begin{array}{l}\text { Design: Intervention study } \\
\text { Age: } 76.4 \text { (1.6) } \\
\text { Quality score: } 25\end{array}$ & $\begin{array}{l}\text { Intervention: } \\
\text { Three weekly sessions of progressive resistance training } \\
\text { Outcome measure: } \\
\text { Class-attendance } \\
\text { Outcome: } \\
\text { Adherence measured through attendance (EX) }\end{array}$ & $\begin{array}{l}\text { Prior exercise adherence }(+) \\
\text { Baseline exercise self-efficacy }(+) \\
\text { Initial general social support }(+) \\
\text { Continued general and program } \\
\text { social support (NS) }\end{array}$ \\
\hline $\begin{array}{l}\text { [35] } \\
\text { Sarkisian et } \\
\text { al. } \\
2007 \\
\text { U.S. }\end{array}$ & $\begin{array}{l}\text { Design: Intervention study } \\
\text { Age: }\left[65^{+}\right] \\
\text {Quality score: } 50\end{array}$ & $\begin{array}{l}\text { Intervention: } \\
\text { Attribution retraining followed by physical activity class } \\
\text { including strength, endurance and flexibility } \\
\text { Outcome measure: } \\
\text { Objective } \\
\text { Pedometer } \\
\text { Outcome: } \\
\text { Weekly step count (PA) }\end{array}$ & Positive age expectations (+) \\
\hline $\begin{array}{l}\text { [18] Shaw } \\
\text { and } \\
\text { Spokane } \\
2008 \\
\text { U.S. }\end{array}$ & $\begin{array}{l}\text { Design: Observational study } \\
\text { Age: }[54-72] \\
\text { Quality score: } 48\end{array}$ & $\begin{array}{l}\text { Intervention: } \\
\text { Not applicable } \\
\text { Outcome measure: } \\
\text { Self-report } \\
\text { Single item, vigorous physical activity or exercise, } 3 \\
\text { times a week or more, over the past } 12 \text { months (PA/ } \\
\text { EX) } \\
\text { Outcome: } \\
\text { Vigorous physical activity or exercise } 3 \text { times a week or } \\
\text { more, over the past } 12 \text { months (yes or no) (PA/EX) }\end{array}$ & $\begin{array}{l}\text { Age }(-) \\
\text { Education }(+) \\
\text { Employment status }(+) \\
\text { Change in employment status }(+) \\
\text { Chronic conditions }(-) \\
\text { Change in chronic conditions } \\
\text { (increase) }(-) \\
\text { General physical functioning }(+) \\
\text { General physical functioning } \\
\text { (increase in limitations)(+) } \\
\text { Depressive symptoms }(-) \\
\text { Change in depressive symptoms } \\
\text { (increase) }(-)\end{array}$ \\
\hline $\begin{array}{l}{[19]} \\
\text { Shimada et } \\
\text { al. } \\
2007 \\
\text { Japan }\end{array}$ & $\begin{array}{l}\text { Design: Observational study } \\
\text { Age: }\left[70^{+}\right] \\
\text {Quality score: } 54\end{array}$ & $\begin{array}{l}\text { Intervention: } \\
\text { Not applicable } \\
\text { Outcome measure: } \\
\text { Self-report } \\
\text { Regular physical activity (yes/no, frequency and nature } \\
\text { of activity: golf, ball games, hiking, home-based or } \\
\text { group exercise, dancing, swimming, martial arts, } \\
\text { jogging, walking, other exercise) } \\
\text { Outcome: } \\
\text { Regular physical activity was defined as carrying out } \\
\text { any type of physical activity } 5 \text { times or more per week } \\
\text { (PA/EX) }\end{array}$ & $\begin{array}{l}\text { Gender (male) }(+) \\
\text { Smoking status }(-) \\
\text { Physical functioning (slow } \\
\text { walking speed) }(-) \\
\text { Fear of falling }(-) \\
\text { Age (NS) } \\
\text { General physical health (NS) }\end{array}$ \\
\hline
\end{tabular}


Table 2 Manuscripts reporting on determinants of physical activity and exercise in healthy older adults (Continued)

\begin{tabular}{llll}
\hline$[20]$ & Design: Observational study & Intervention: & Prior exercise adherence $(+)$ \\
Stiggelbout & Age: $60.9(8.4)$ & Not applicable & Perceived quality of the progra \\
et al. & Quality score: 50 & Outcome measure: & $(+)$ \\
2006 & & Self-report & Exercise attitude $(+)$
\end{tabular}

$2006 \quad$ Self-report

The

the Dutch Monitor on Physical Activity and

Netherlands

Health to assess compliance with Dutch public-health guidelines

Outcome:

Perceived quality of the program

$(+)$

Exercise attitude $(+)$

Exercise barriers (-)

Norm-active is defined as performing 30 minutes or more of moderate-intensity physical activity on most, and preferably all, days - either in a single session or

Exercise intention $(+)$ Exercise self-efficacy (NS)

Coping (NS)

accumulated in multiple bouts of al least 10 minutes (PA/EX)

\begin{tabular}{ll}
\hline 21$]$ Touvier & Design: Observational study \\
et al. & Age:[45-64] \\
2010 & Quality score:46 \\
France &
\end{tabular}

Intervention:

Not applicable

Outcome measure:

Self-report

MAQ Past 12 month physical activity during leisure

time and at work

Outcome:

Subjects were considered to meet overall PA

recommendations if their overall PA was $\geq 60$ min per

week of vigorous activities with at least $20 \mathrm{~min}$ per

session or $\geq 150$ min per week of moderate activities (PA/EX)

\begin{tabular}{ll}
\hline 22] Tu et & Design: Observational study \\
al. & Age: $63.7(7.3)$ \\
2004 & Quality score: 52 \\
US &
\end{tabular}

\section{Intervention:}

Not applicable

Outcome measure:

Class-attendance recorded by research assistant

Outcome:

Measured attendance (EX)

Social influences and support

[36] Wilcox

and King

2004

U.S.
Design: Intervention study

Age: 70.2 (4.1)

Quality score: 25

\section{Intervention:}

Exercise classes aerobic and strength training or stretching and relaxation combined with home-work with telephone counselling to encourage participation in the program

\section{Outcome measure:}

Self-report

Exercise logs validation through class-attendance and

'vitalogs'

Outcome:

Participation calculated as percentage of exercise sessions completed divided by sessions prescribed (EX)

[37] Design: Intervention study

Williams

and Lord

1995

Age: 71.6 (5.5)

Quality score: 16

Australia

\section{Intervention:}

Exercise to improve balance, coordination, strength and cardiorespiratory fitness

Outcome measure:

Class-attendance and activities outside the program

Outcome:

Adherence was defined as number of exercise classes attended (EX)

\section{Age (-)}

Reaction time $(+)$

Psychoactive drug use (-)

Physical strength $(+)$

Cognitive reasoning ability $(+)$

Depression (-)

Self reported general physical health outcome realizations (+)

Self reported physical functioning outcome realizations (+)

Self reported cognitive functioning outcome realizations $(+)$

Self reported psychological outcome realizations (+)

Education (NS)

BMI (NS)

General physical health (NS) General mental health (NS)

General cognitive functioning (NS)

Baseline activity level (NS)

Locus of control (NS) 
Table 2 Manuscripts reporting on determinants of physical activity and exercise in healthy older adults (Continued)

\begin{tabular}{llll}
\hline$[23]$ & Design: Observational study & Intervention: & Age $(-)$ \\
Yasunaga et & Age: [65-83] & Not applicable & Gender (male) $(+)$ \\
al. & Quality score: 88 & Outcome measure: & Weather conditions $(g o 0 d)(+)$ \\
2008 & & Objective & \\
Japan & Pedometer/accelerometer & Outcome: \\
& Number of steps taken and the intensity of physical \\
& activity (PA) & \\
\hline
\end{tabular}

* Cluster of multiple manuscripts reporting on the same original data set

Table 3 Methodological quality of the included studies

\begin{tabular}{|c|c|c|c|c|c|c|c|c|c|c|}
\hline \multirow[b]{2}{*}{ Reference } & & \multicolumn{8}{|c|}{ Item* } & \multirow[t]{2}{*}{ Quality score $\%$} \\
\hline & & 1 & 2 & 3 & 4 & 5 & 6 & 7 & 8 & \\
\hline Boyette et al., 1997 & & $?$ & + & + & - & 0.17 & 0.17 & - & - & 29 \\
\hline Brassington et al., 2002 & & $?$ & + & $?$ & - & 0 & 0 & + & + & 38 \\
\hline Burton et al., 1999 & & $?$ & - & + & - & 0.45 & 0.45 & + & + & 49 \\
\hline Cheung et al., 2007 & & $?$ & + & $?$ & - & 0 & 0 & + & - & 25 \\
\hline Costanzo and Walker 2008 & & + & + & $?$ & - & 0 & 0 & + & + & 50 \\
\hline \multirow[t]{4}{*}{ Cluster $1^{* *}$} & Garcia and King, 1991 & $?$ & $?$ & $?$ & - & 0.69 & 0.62 & + & + & 41 \\
\hline & King et al., 1997 & $?$ & $?$ & $?$ & - & 0.6 & 0.6 & + & + & 40 \\
\hline & Oka et al., 1995 & - & $?$ & $?$ & - & 0.33 & 0.33 & + & - & 21 \\
\hline & Oman and King, 1998 & - & $?$ & $?$ & - & 0.5 & 0.5 & + & + & 38 \\
\hline \multirow[t]{2}{*}{ Cluster $2^{* *}$} & McAuley et al., 2003 & $?$ & + & $?$ & - & 0 & 0 & + & + & 38 \\
\hline & McAuley et al., 2007 & $?$ & + & $?$ & - & 0.7 & 0.8 & + & + & 56 \\
\hline Dubbert et al., 2002 & & $?$ & + & $?$ & - & 0.14 & 0.14 & + & + & 41 \\
\hline Emery et al., 1992 & & $?$ & + & $?$ & - & 0.25 & 0.25 & - & - & 19 \\
\hline Finkelstein et al., 2008 & & $?$ & + & $?$ & + & 1 & 1 & + & - & 63 \\
\hline Hirvensalo et al., 2000 & & $?$ & $?$ & $?$ & - & 0.25 & 0.25 & + & $?$ & 19 \\
\hline Jancey et al., 2007 & & - & - & - & - & 0.71 & 0.43 & + & + & 39 \\
\hline Kahana et al., 2005 & & $?$ & $?$ & $?$ & - & 0.50 & 0.50 & + & + & 38 \\
\hline Li et al., 2001 & & $?$ & - & - & - & 0 & 0 & + & + & 25 \\
\hline Li et al., 2005 & & $?$ & + & $?$ & - & 0.43 & 0.29 & + & + & 47 \\
\hline Lucidi et al., 2006 & & + & + & $?$ & - & 0 & 0 & + & + & 50 \\
\hline Michael et al., $2010^{\S}$ & & $?$ & + & + & - & 1 & 1 & + & + & 75 \\
\hline Morey et al., 2003 & & - & + & $?$ & - & 0.29 & 0.29 & + & - & 32 \\
\hline Nitz and Choy, 2007 & & $?$ & + & $?$ & - & 0.09 & 0.09 & - & - & 15 \\
\hline Oman and King, 2000 & & $?$ & + & $?$ & - & 0 & 0 & + & - & 25 \\
\hline Rhodes et al., 2001 & & $?$ & + & $?$ & - & 0 & 0 & + & - & 25 \\
\hline Sarkisian et al., 2007 & & $?$ & + & $?$ & + & 0 & 0 & + & + & 50 \\
\hline Shaw and Spokane, 2008 & & + & $?$ & $?$ & - & 0.40 & 0.40 & + & + & 48 \\
\hline Shimada et al., 2007 & & + & - & $?$ & - & 0.67 & 0.67 & + & + & 54 \\
\hline Stiggelbout et al., 2006 & & + & + & $?$ & - & 0 & 0 & + & + & 50 \\
\hline Touvier et al., 2010 & & $?$ & $?$ & $?$ & - & 0.67 & 1 & + & + & 46 \\
\hline Tu et al., 2004 & & $?$ & $?$ & $?$ & - & 0.56 & 0.56 & + & + & 39 \\
\hline Wilcox and King, 2004 & & $?$ & + & $?$ & - & 0 & 0 & + & - & 25 \\
\hline Williams and Lord, 1995 & & $?$ & - & $?$ & - & 0.12 & 0.12 & + & - & 16 \\
\hline Yasunaga et al., 2008 & & $?$ & + & + & + & 1 & 1 & + & + & 88 \\
\hline
\end{tabular}

* Item number corresponds with item description in Table 1

** Cluster of multiple manuscripts reporting on the same original data set

$\S$ Manuscripts with a percentage of $70 \%$ or more were considered high quality 
measured with a reliable and valid tool). The methodological quality score ranged from 15 to $88 \%$. Two studies received scores above 70\%; a score considered to indicate "high" quality $[16,23]$.

\section{Determinants of PA}

Supported by one high quality study [23] and one low quality study [12], moderate evidence was found for a positive association between male gender and PA, as well as younger age and PA. For all other possible determinants of PA we found insufficient evidence.

\section{Determinants of EX}

Based on four low quality studies [22,24,28,31,42] (two manuscripts reported on the same study), we found no evidence for an association between age and EX. Based on three low quality studies we found no evidence for a relation between ethnicity and EX [22,31,42].

Based on three low quality studies, we found no relation between general physical health and EX [22,31,37]. Four low quality studies provided moderate evidence for a positive association between general physical functioning and EX [28,31]. Moderate evidence for a negative association between (chronic) conditions and diseases and EX was observed [22,31]. Based on two low quality studies moderate evidence was found for a negative association between depression and EX [31,37]. We found moderate evidence for a positive association of prior exercise adherence [31,32] and self-reported beneficial health or physical functioning outcomes $[25,37]$ with EX. The four studies supporting this evidence were of low methodological quality.

Moderate evidence, based on two low quality studies, was found for a positive association between change in exercise self-efficacy and EX [25,43]. Based on two low quality studies we found no evidence for an association between locus of control and EX [31,37]. Major life events were reported in two low quality studies [33,36]. Based on these studies, moderate evidence was found for a negative association between major life events and EX. For all other determinants assessed, insufficient evidence was found.

\section{Determinants of PA/EX}

Based on seven low quality studies [14,17,19,26,27,41], we found no evidence for an association between age and PA/EX. Based on two low quality studies, we found moderate evidence for a negative association between $B M I$ and PA/EX [17,27]. Supported by two low quality studies we found no evidence for an association between general physical health and PA/EX [14,19]. We found moderate evidence for a positive association between baseline activity (based on two low quality studies; $[17,27]$ and PA/EX.

We found no evidence for an association between baseline social support and PA/EX, based on two low quality studies $[20,44]$. For all other possible determinants of PA/EX we found insufficient evidence.

\section{Discussion}

The aim of this review was to assess the determinants of physical activity (PA) as well as exercise (EX) in healthy older adults, considering the methodological quality of the included studies. The 34 manuscripts included in this review provide an overview of the determinants of PA or EX that have been studied in the last 20 years. Although we set out to differentiate between determinants of PA and EX, we concluded "insufficient evidence" for most associations between possible determinants and PA or EX. This was mainly due to multiple low quality studies reporting inconsistent findings, lack of high quality studies, and often only one manuscript reporting on a particular determinant. The limited evidence available on PA and EX revealed a dissimilarity concerning the determinant age; moderate evidence was found for a positive association with younger age and PA, whereas no evidence was found for an association of age and EX. No inverted associations were found between determinants of PA and EX. No further illustration could be made of similarities or dissimilarities between PA and EX. This emphasises the need for additional research, particularly on determinants of PA.

We have categorised PA and EX according to the definitions by Caspersen et al. [8]. These definitions have been adopted by the ACSM [3]. In addition to the possible differences in (determinants of) PA versus EX, differences within PA and EX categories may exist. Indeed, we have included four manuscripts reporting on PA in which different behavioural outcomes were assessed (step count, walking, gardening, heavy housework, neighbourhood walking and engaging in activities with neighbours). As of yet, there is too little high-quality research to differentiate within activity categories. In future research, more differentiation is desirable. Not withstanding these methodological issues, the renewed focus on PA (as opposed to EX) could be important since recent work on the (cost-) effectiveness of PA and EX interventions showed the long term beneficial effect of PA over EX. In many behavioural-based lifestyle interventions, participants are taught to integrate PA in their daily lives [46-48]. Especially activities such as walking, gardening or housework could be very well integrated in the lives of older adults.

Overall, the methodological quality of the included studies was low, with only two manuscripts scoring above $70 \%$ (high quality). Determinants were often measured by different instruments, complicating comparison between studies. Moreover, most of the determinants were assessed using instruments with unknown or poor reliability and validity. The most commonly used 
instruments assessing PA or EX behaviour were selfreport measures. Unfortunately, there are currently no self-report instruments to assess PA or EX in older adults that are both reliable and valid [49]. Instruments like pedometers and accelerometers, which generally perform better in terms of reliability and validity [50-53], are therefore increasingly used to assess PA or EX $[54,55]$. Applying a strict methodological quality assessment could have led to overly cautious conclusions on the evidence in the field. Although the quality assessment is one of many ways to judge the strength of evidence, and has its own methodological restrictions, we do feel that future research on determinants of PA and EX in older adults could be greatly improved.

We recommend additional research on the determinants of PA. We further recommend the development and use of valid and reliable measurement instruments for determinants as well as the use of objective measurement instruments of PA and EX. The use of measurement instruments with good psychometric properties and the use of comparable, and preferably similar, instruments assessing determinants should allow for a better comparison between studies.

Future interventions should carefully build upon the limited evidence available. Some of the assessed determinants (e.g. gender and age) suggest the targeting of subgroups within the population for interventions. The Intervention Mapping protocol [56] provides guidelines and tools for theory based development of programme materials and activities to increase physical activity and exercise. This approach can be used to systematically explain and change modifiable determinants of PA or EX (such as exercise self-efficacy).

In our review we have identified many determinants of PA and EX in healthy older adults (demographic determinants; determinants of health and health behaviour; psychological determinants; social determinants; environmental determinants; determinants related to the intervention). However, there is evidence available on possible other determinants of physical activity and exercise behaviour that we could not identify in our search. These include genetic determinants [57-59], policy related determinants $[60,61]$ and, in addition to determinants of planned behaviour change[62], unplanned or unintentional behavioural change [63-67]. Recent work has shown the possibilities of trans-disciplinary research integrating theoretical approaches (e.g. genetic, physiological, psychological) in physical activity research that could provide a framework for further research $[68,69]$.

Limitations inherent to writing a review consist of "publication bias" and the possibility that manuscripts may have been overlooked. We have tried to prevent the latter by selecting a medical and a psychological database, using broad search terms and checking reference lists from other reviews. Another limitation, specific to this review, is the selection of literature describing studies with a population aged 55 and older. Looking at this relatively broad age range combined, may have masked possible differences in determinants between subsamples of this age range. Additionally, excluding specific subsamples of the older population (i.e. confined geographic area or diagnosed diseases) may have further masked possible differences in determinants between subsamples.

\section{Conclusions}

A large number of determinants of PA and EX were examined and for most determinants there was insufficient evidence. Assessed determinants of older adults' PA, EX and PA/EX reported in this review, such as age, BMI, exercise self-efficacy and social support, are similar to determinants reported by Trost et al. [70] for the entire adult population. Furthermore, they also resemble the determinants of initiation and maintenance of physical activity among older adults reported by Van Stralen et al. [7]. Unlike these earlier reviews we took the methodological quality of the manuscripts into account. This resulted in more cautious conclusions on the available evidence in the field.

Although a diverse set of possible determinants occurs in the literature (e.g., characteristics of the individual, of the social and physical environment and of the intervention), other possible determinants remain largely unstudied. There is a relative shortage of manuscripts assessing determinants of PA, which needs to be addressed in future research, ideally using objective, valid and reliable measures. Subsequently, the possible differences in determinants between PA and EX need further study as well.

\section{Acknowledgements}

The authors gratefully acknowledge the Dutch Ministry of Health, Welfare and Sport for financial support of the contributions of MAK, MWV and MHR. The authors thank Rebecca Kuiper for her help in the appraisal of the methodological quality of the studies.

\section{Author details}

'Body@Work, Research Center for Physical Activity, Work and Health, TNO-VU University Medical Center, Amsterdam, The Netherlands. ${ }^{2}$ Department of Public and Occupational Health, $\mathrm{EMGO}^{+}$Institute for Health and Care Research, VU University Medical Center, Amsterdam, The Netherlands. ${ }^{3}$ TNO, Leiden, The Netherlands.

\section{Authors' contributions}

MK and MV conducted the literature search. All authors evaluated the appropriateness of included manuscripts. All authors contributed equally to the editing and approving of the final version of the paper.

\section{Authors' information}

Margot Koeneman, MSc, is a PhD student at Body@Work research center, a joint initiative of TNO, Leiden, The Netherlands and VU University, and VU 
University Medical Centre, Amsterdam, The Netherlands. Her background is in Social Psychology. She previously worked as a junior researcher at the AMC medical centre, Amsterdam.

Dr Marieke Verheijden is working at TNO (Netherlands organisation for applied scientific research) in Leiden. Her professional background is in Nutrition and Health. Most of her work was on the development, implementation and evaluation of (tailored) health promotion programs, predominantly in the areas of prevention of overweight by promoting healthy nutrition and physical activity. She is also a staff member of the Body@Work research centre Physical activity, Work and Health TNO VU medical centre.

Dr Mai Chin A Paw is associate professor at the Department of Public and Occupational Health, EMGO Institute - VU University Medical Centre in Amsterdam, The Netherlands. Her background is in Human Movement Science and Epidemiology. She obtained her PhD in 1999 for her thesis that investigated the effects of physical exercise and micronutrient

supplementation on the health of frail older people. Currently, she chairs the section Youth and Health within the department of Public and Occupational health and is involved in several research projects. Examples are the development and evaluation of strategies promoting physical activity and reducing sedentary behaviours, measurement of physical activity, and prevention and treatment of obesity. She is associate editor of The Journal of Science and Medicine in Sport and member of the editorial board of the International Journal of Behavioral Nutrition and Physical Activity. Professor Dr Marijke Hopman-Rock is working at TNO (Netherlands organisation for applied scientific research) in Leiden and professor in Physical activity and health of older persons at the VU university medical centre in Amsterdam. She is also in the management team of the Body@Work research centre Physical activity, Work and Health TNO VU medical centre. Her professional background is in Biology, Psychology (statistics) and Epidemiology (PhD). As a part-time professor in Amsterdam she supervises several PhD students. She is also past (founding) chair of the European Network for Action on Ageing and Physical Activity (EUNAAPA) and associate partner in several EU projects in the area of physical activity. She is associate editor of the Journal of Aging and Physical Activity (JAPA) and BMC Public Health and is also on the board of other journals, including the Human Kinetics journal Active Aging Today. She is a member of the TOP ZonMw/NWO committee. In the past she was Head of the Department of PA and Health at TNO and program manager in the same area; Vicepresident of the EGREPA (European group for Research in Elderly and PA) and co-chair of the Aging interest group of the American College of Sport and Exercise Medicine (ACSM).

\section{Competing interests}

The authors declare that they have no competing interests.

Received: 23 May 2011 Accepted: 28 December 2011 Published: 28 December 2011

\section{References}

1. Nelson ME, Rejeski WJ, Blair SN, Duncan PW, Judge JO, King AC, Macera CA, Castaneda-Sceppa C: Physical activity and public health in older adults: recommendation from the American College of Sports Medicine and the American Heart Association. Med Sci Sports Exerc 2007, 39:1435-1445.

2. Singh MA: Exercise comes of age: rationale and recommendations for a geriatric exercise prescription. J Gerontol A Biol Sci Med Sci 2002, 57 : M262-M282.

3. Chodzko-Zajko WJ, Proctor DN, Fiatarone Singh MA, Minson CT, Nigg CR, Salem GJ, Skinner JS: American College of Sports Medicine position stand. Exercise and physical activity for older adults. Med Sci Sports Exerc 2009, 41:1510-1530.

4. Ecclestone NA, Myers AM, Paterson DH: Tracking older participants of twelve physical activity classes over a three-year period. J Aging Phys Act 1998, 6:70-82

5. Stiggelbout M, Hopman-Rock M, Tak E, Lechner L, van MW: Dropout from exercise programs for seniors: a prospective cohort study. J Aging Phys Act 2005, 13:406-421.

6. Martin KA, Sinden AR: Who Will Stay and Who Will Go? A Review of Older Adults' Adherence to Randomized Controlled Trials of Exercise. J Aging Phys Act 2001, 9:91-114.
7. van Stralen MM, de Vries H, Mudde AN, Bolman C, Lechner L: Determinants of initiation and maintenance of physical activity among older adults: a literature review. Health Psychol Review 2009, 3:147-207.

8. Caspersen CJ, Powell KE, Christenson G: Physical activity, exercise and physical fitness: definitions and distinctions for health-related research. Public Health Rep 1985, 100:126-131.

9. Chinapaw MJ, Proper KI, Brug J, van MW, Singh AS: Relationship between young peoples' sedentary behaviour and biomedical health indicators: a systematic review of prospective studies. Obes Rev 2011, 12:e621-e632.

10. Brown H, Hume C, ChinAPaw M: Validity and reliability of instruments to assess potential mediators of children's physical activity: A systematic review. J Sci Med Sport 2009, 12:539-548.

11. Uijtdewilligen L, Nauta J, Singh AS, van MW, Twisk JW, van der HK, Chinapaw MJ: Determinants of physical activity and sedentary behaviour in young people: a review and quality synthesis of prospective studies. Br J Sports Med 2011, 45:896-905.

12. Burton LC, Shapiro S, German PS: Determinants of physical activity initiation and maintenance among community-dwelling older persons. Prev Med 1999, 29:422-430.

13. Hirvensalo M, Lintunen T, Rantanen $\mathrm{T}$ : The continuity of physical activity-a retrospective and prospective study among older people. Scand J Med Sci Sports 2000, 10:37-41.

14. Kahana E, Kahana B, Zhang J: Motivational Antecedents of Preventive Proactivity in Late Life: Linking Future Orientation and Exercise. Motiv Emot 2005, 29:438-459.

15. Li F, Fisher J, Brownson RC: A multilevel analysis of change in neighborhood walking activity in older adults. J Aging Phys Act 2005, 13:145-159.

16. Michael YL, Perdue LA, Orwoll ES, Stefanick ML, Marshall LM: Physical Activity Resources and Changes in Walking in a Cohort of Older Men. Am J Public Health 2010, 100:654-660.

17. Nitz JC, Choy NL: Changes in activity level in women aged $40-80$ years. Climacteric 2007, 10:408-415.

18. Shaw BA, Spokane LS: Examining the association between education level and physical activity changes during early old age. J Aging Health 2008, 20:767-787.

19. Shimada H, Lord SR, Yoshida H, Kim H, Suzuki T: Predictors of cessation of regular leisure-time physical activity in community-dwelling elderly people. Gerontology 2007, 53:293-297.

20. Stiggelbout M, Hopman-Rock M, Crone M, Lechner L, van MW: Predicting older adults' maintenance in exercise participation using an integrated social psychological model. Health Educ Res 2006, 21:1-14.

21. Touvier M, Bertrais S, Charreire H, Vergnaud AC, Hercberg S, Oppert JM: Changes in leisure-time physical activity and sedentary behaviour at retirement: a prospective study in middle-aged French subjects. Int $\rfloor$ Behav Nutr Phys Act 2010, 7:14.

22. Tu W, Stump TE, Damush TM, Clark DO: The effects of health and environment on exercise-class participation in older, urban women. $J$ Aging Phys Act 2004, 12:480-496.

23. Yasunaga A, Togo F, Watanabe E, Park H, Park S, Shephard RJ, Aoyagi Y: Sex, age, season, and habitual physical activity of older Japanese: the Nakanojo study. J Aging Phys Act 2008, 16:3-13.

24. Boyette LW, Sharon BF, Brandon LJ: Exercise adherence for a strength training program in older adults. J Nutr Health Aging 1997, 1:93-97.

25. Brassington GS, Atienza AA, Perczek RE, DiLorenzo TM, King AC: Intervention-related cognitive versus social mediators of exercise adherence in the elderly. Am J Prev Med 2002, 23:80-86.

26. Emery CF, Hauck ER, Blumenthal JA: Exercise adherence or maintenance among older adults: 1-year follow-up study. Psychol Aging 1992, 7:466-470.

27. Jancey J, Lee A, Howat P, Clarke A, Wang K, Shilton T: Reducing attrition in physical activity programs for older adults. J Aging Phys Act 2007, 15:152-165.

28. King AC, Kiernan M, Oman RF, Kraemer HC, Hull M, Ahn D: Can we identify who will adhere to long-term physical activity? Signal detection methodology as a potential aid to clinical decision making. Health Psychol 1997, 16:380-389.

29. Lucidi F, Grano C, Barbaranelli C, Violani C: Social-cognitive determinants of physical activity attendance in older adults. J Aging Phys Act 2006, 14:344-359. 
30. McAuley E, Morris KS, Motl RW, Hu L, Konopack JF, Elavsky S: Long-term follow-up of physical activity behavior in older adults. Health Psychol 2007, 26:375-380.

31. Morey MC, Dubbert PM, Doyle ME, MacAller H, Crowley GM, Kuchibhatla M, Schenkman M, Horner RD: From Supervised to Unsupervised Exercise: Factors Associated with Exercise Adherence. J Aging Phys Act 2003, 11:351-368.

32. Oman RF, King AC: Predicting the adoption and maintenance of exercise participation using self-efficacy and previous exercise participation rates. Am J Health Promot 1998, 12:154-161

33. Oman RF, King AC: The effect of life events and exercise program format on the adoption and maintenance of exercise behavior. Health Psychol 2000, 19:605-612.

34. Rhodes RE, Martin AD, Taunton JE: Temporal relationships of self-efficacy and social support as predictors of adherence in a 6-month strengthtraining program for older women. Percept Mot Skills 2001, 93:693-703.

35. Sarkisian CA, Prohaska TR, Davis C, Weiner B: Pilot test of an attribution retraining intervention to raise walking levels in sedentary older adults J Am Geriatr Soc 2007, 55:1842-1846.

36. Wilcox S, King AC: The effects of life events and interpersonal loss on exercise adherence in older adults. J Aging Phys Act 2004, 12:117-130.

37. Williams $P$, Lord SR: Predictors of adherence to a structured exercise program for older women. Psychol Aging 1995, 10:617-624

38. Cheung C, Wyman J, Gross C, Peters J, Findorff M, Stock H: Exercise behavior in older adults: a test of the transtheoretical model. J Aging Phys Act 2007, 15:103-118.

39. Costanzo C, Walker SN: Incorporating self-efficacy and interpersonal support in an intervention to increase physical activity in older women. Women Health 2008, 47:91-108.

40. Dubbert PM, Cooper KM, Kirchner KA, Meydrech EF, Bilbrew D: Effects of nurse counseling on walking for exercise in elderly primary care patients. J Gerontol A Biol Sci Med Sci 2002, 57:M733-M740.

41. Finkelstein EA, Brown DS, Brown DR, Buchner DM: A randomized study of financial incentives to increase physical activity among sedentary older adults. Prev Med 2008, 47:182-187.

42. Garcia AW, King AC: Predicting Long-term Adhernce to Aerobic Exercise: A Comparison of Two Models. J Sport Exercise Psy 1991, 13:394-410.

43. Li F, McAuley E, Harmer P, Duncan TE, Chaumeton NR: Tai Chi Enhances Self-Efficacy and Exercise Behavior in Older Adults. J Aging Phys Act 2001, 9:161-171

44. McAuley E, Jerome GJ, Elavsky S, Marquez DX, Ramsey SN: Predicting longterm maintenance of physical activity in older adults. Prev Med 2003, 37:110-118.

45. Oka RK, King AC, Young DR: Sources of social support as predictors of exercise adherence in women and men ages 50 to 65 years. Womens Health 1995, 1:161-175.

46. Opdenacker J, Boen F, Coorevits N, Delecluse C: Effectiveness of a lifestyle intervention and a structured exercise intervention in older adults. Prev Med 2008, 46:518-524.

47. Sevick MA, Dunn AL, Morrow MS, Marcus BH, Chen GJ, Blair SN: Costeffectiveness of lifestyle and structured exercise interventions in sedentary adults. Results of project ACTIVE. Am J Prev Med 2000, 19:1-8.

48. Van Roie E, Delecluse C, Opdenacker J, De BK, Kennis E, Boen F: Effectiveness of a lifestyle physical activity versus a structured exercise intervention in older adults. J Aging Phys Act 2010, 18:335-352.

49. Forsen L, Loland NW, Vuillemin A, Chinapaw MJ, van Poppel MN, Mokkink LB, van MW, Terwee CB: Self-administered physical activity questionnaires for the elderly: a systematic review of measurement properties. Sports Med 2010, 40:601-623.

50. Harris TJ, Owen CG, Victor CR, Adams R, Ekelund U, Cook DG: A comparison of questionnaire, accelerometer, and pedometer: Measures in older people. Med Sci Sports Exerc 2009, 41:1392-1402.

51. Shephard RJ: Limits to the measurement of habitual physical activity by questionnaires. Br J Sports Med 2003, 37:197-206.

52. Hawkins MS, Storti KL, Richardson CR, King WC, Strath SJ, Holleman RG Kriska AM: Objectively measured physical activity of USA adults by sex, age, and racial/ethnic groups: a cross-sectional study. Int J Behav Nutr Phys Act 2009, 6:31.

53. Mathie MJ, Coster AC, Lovell NH, Celler BG: Accelerometry: providing an integrated, practical method for long-term, ambulatory monitoring of human movement. Physiol Meas 2004, 25:R1-20.
54. de Bruin ED, Hartmann A, Uebelhart D, Murer K, Zijlstra W: Wearable systems for monitoring mobility-related activities in older people: $A$ systematic review. Clin Rehabil 2008, 22:878-895.

55. Tudor-Locke C, Hart TL, Washington TL: Expected values for pedometerdetermined physical activity in older populations. Int I Behav Nutr Phys Act 2009, 6:59

56. Bartholomew LK, Parcel GS, Kok G, Gottlieb NH: Planning health promotion programs. An intervention mapping approach. Third edition. San Francisco: Jossey-Bass; 2011.

57. Beunen $\mathrm{G}$, Thomis M: Genetic determinants of sports participation and daily physical activity. Int J Obes 1999, 23.

58. De Moor MHM, Posthuma D, Hottenga JJ, Willemsen G, Boomsma DI, De Geus EJC: Genome-wide linkage scan for exercise participation in Dutch sibling pairs. Eur J Hum Genet 2007, 15:1252-1259.

59. Frederiksen $H$, Christensen $\mathrm{K}$ : The influence of genetic factors on physical functioning and exercise in second half of life. Scand J Med Sci Sports 2003, 13:9-18.

60. Eyler A, Brownson R, Schmid T, Pratt M: Understanding Policies and Physical Activity: Frontiers of Knowledge to Improve Population Health. J Phys Activ Health 2010, 7:S9-S12

61. Scott F, Young A, Dinan-Young S, Harding M, Lewis S, Fisken S: EUNAAPA Work Package 5. Expert Survey on Physical Activity Programmes and Physical Activity Promotion Strategies for Older People.[http://www. eunaapa.org/media/cross-national_report_expert_survey_on_pa_ programmes_and_promotion_strategies_2008_pdf].

62. Ajzen I: The theory of planned behaviour. Organ Behav Hum Dec 1991 50:179-211.

63. Aarts H, Dijksterhuis A: Habits as knowledge structures: automaticity in goal-directed behavior. J Pers Soc Psychol 2000, 78:53-63.

64. Berry T, Spence JC: Automatic activation of exercise and sedentary stereotypes. Res Q Exerc Sport 2009, 80:633-640.

65. Berry TR: Who's even interested in the exercise message? attentional bias for exercise and sedentary-lifestyle related words. J Sport Exerc Psychol 2006, 28:4-17.

66. Dijksterhuis A, Bos MW, Nordgren LF, van Baaren RB: On making the right choice: the deliberation-without-attention effect. Science 2006, 311:1005-1007.

67. Kremers SP, de Bruijn GJ, Visscher TL, van MW, de Vries NK, Brug J: Environmental influences on energy balance-related behaviors: a dualprocess view. Int J Behav Nutr Phys Act 2006, 3:9.

68. Bryan A, Hutchison KE, Seals DR, Allen DL: A transdisciplinary model integrating genetic, physiological, and psychological correlates of voluntary exercise. Health Psychol 2007, 26:30-39.

69. Epstein LH: Integrating theoretical approaches to promote physical activity. Am J Prev Med 1998, 15:257-265.

70. Trost SG, Owen N, Bauman AE, Sallis JF, Brown W: Correlates of adults' participation in physical activity: review and update. Med Sci Sports Exerc 2002, 34:1996-2001.

doi:10.1186/1479-5868-8-142

Cite this article as: Koeneman et al: Determinants of physical activity and exercise in healthy older adults: A systematic review. Internationa Journal of Behavioral Nutrition and Physical Activity 2011 8:142.

\section{Submit your next manuscript to BioMed Central and take full advantage of:}

- Convenient online submission

- Thorough peer review

- No space constraints or color figure charges

- Immediate publication on acceptance

- Inclusion in PubMed, CAS, Scopus and Google Scholar

- Research which is freely available for redistribution 